\title{
Contribution à l'inventaire et à la domestication des espèces alimentaires sauvages de Côte d'Ivoire: Cas des Départements d'Agboville et d'Oumé
}

\author{
1DJAHA Akadjé Jean Baptiste, ${ }^{2}$ GNAHOUA Guy Modeste \\ ${ }^{1}$ Centre National de Recherche Agronomique, Station de Lataha, BP 856 Korhogo, Côte d'Ivoire \\ ${ }^{2}$ Centre National de Recherche Agronomique, Station Technologie, 08 BP 881 Abidjan, Côte d'Ivoire \\ Auteur correspondant : gm.gnahoua@hotmail.fr
}

Original submitted in on $6^{\text {th }}$ May 2014. Published online at www.m.elewa.org on $30^{\text {th }}$ June 2014. http://dx.doi.org/10.4314/iab.v78i1.8

\section{RESUME}

Objectif : L'objectif de la présente étude était de déterminer les espèces alimentaires sauvages encore présentes dans le département d'Agboville et de sauvegarder en collections celles menacées de disparition et ayant un intérêt communautaire avéré.

Méthodologie et résultats : Une enquête ethnobotanique a été effectuée. Des semences et des sauvageons ont servi à produire des plants (en pépinière) qui ont été mis en collection. L'enquête a révélé 18 espèces réparties en 18 genres et 15 familles. Les arbres représentent $81,8 \%$ de la flore inventoriée et les espèces à graines ou amandes comestibles, $50 \%$. En pépinière, les taux de levée ont varié de 60 à $100 \%$, sauf chez Garcinia kola et Xylopia aethiopica. En collection, les 4 espèces retenues, âgées de 7 ans, ont affiché des taux de survie de 40,71 à $86 \%$ et des hauteurs de 4,86 à $12,68 \mathrm{~m}$.

Conclusion et application : Avec seulement 18 espèces recensées en zone de forêt, l'enquête a révélé le niveau de dégradation avancée de la végétation des sites prospectés. Les taux de survie élevés en pépinière montrent que ces espèces peuvent se reproduire aussi bien par graines que par sauvageons. La conduite de sauvageons en pépinière peut aider à résoudre les problèmes liés à la rareté saisonnière des semences. Les bonnes aptitudes à la régénération en milieu contrôlé (pépinière) et à la conduite en peuplement indiquent que ces essences peuvent croître dans les systèmes agroforestiers traditionnels (association avec les cultures de rente et les cultures vivrières). Toutefois, il importe d'une part d'appliquer des prétraitements aux semences pour lever plus rapidement leur dormance et d'autre part d'envisager la multiplication végétative des espèces majeures pour hâter leur entrée en production et susciter un plus grand intérêt des populations rurales.

Mots-clés : espèces alimentaires sauvages, enquête ethnobotanique, domestication

Contribution to the inventory and domestication of wild edible species of Côte d'lvoire: Case of Agboville and Oumé Departments

\section{ABSTRACT}

Objective: The current study aims at identifying the wild edible species still present in the department of Agboville and save in collections the endangered ones that have a high communitary importance.

Methodology and Results: An ethnobotanical survey was conducted. Seeds and wildlings collected were used to produce plants (in nursery) and create a collection. The survey revealed 18 species distributed in 18 genera 
and 15 families. Trees represent $81.8 \%$ of the surveyed flora and species producing edible seeds or kernels were $50 \%$. In nursery, the emergence rate of species ranged between 60 and 100\%, except for Garcinia kola and Xylopia aethiopica. In collections, at 7 years, the 4 main species assessed showed survival rates of 40.71 to $86 \%$ and heights of 4.86 to $12.68 \mathrm{~m}$.

Conclusion and Application: With only 18 species recorded in a forest area, the study revealed the high level of vegetation degradation in the surveyed sites. This shows the need for an urgent domestication program of residual species before total extinction. The high survival rates observed in nursery showed that the studied species can be propagated by seeds and by wildlings as well. Propagation by wildlings can help solve the problems of seeds seasonal scarcity. The satisfactory skills of the studied species in nursery and plantation indicate that they are suited to grow in traditional agroforestry systems, in association with cash crops (coffee and cocoa) and food crops. Nevertheless, it is important to practice seed pretreatments to remove dormancy faster. Also, vegetative propagation of priority species should be undertaken to shorten unproductive period in order to raise up a greater interest from rural populations.

Key words: wild edible species, ethnobotanical survey, domestication.

\section{INTRODUCTION}

Les plantes constituent des ressources naturelles indispensables à l'alimentation et aux soins de l'homme (Dupriez et De Leener, 1993). Cependant, de nombreuses espèces végétales ont disparu ou se sont raréfiées, essentiellement durant ce dernier siècle, du fait de l'action anthropique (exploitation forestière, expansion agricole, urbanisation, etc.). En Côte d'Ivoire, la dégradation du couvert forestier a été rapide et excessive; environ 12 millions d'hectares ont disparu entre 1960 et 1990, entraînant la raréfaction et la disparition de plusieurs espèces alimentaires et médicinales (Kouamé et Gnahoua, 2008). Les espèces fruitières sauvages contribuent à la sécurité alimentaire des populations rurales en particulier et font l'objet d'un commerce florissant dont les principaux acteurs sont les femmes. Les espèces fruitières alimentaires et médicinales de Côte d'Ivoire ont fait l'objet d'études de base, en botanique (Aubreville, 1959) et en ethnobotanique (Gauthier-Béguin, 1992; Ambé,

\section{MATERIEL ET METHODES}

Sites de l'étude : Deux zones géographiques ont servià la conduite des travaux de la présente étude (Figure 1): les Départements d'Agboville (Sud-Est) et d'Oumé (Centre-Ouest). Les enquêtes ont été conduites dans cinq (5) localités du Département d'Agboville (GrandYapo, Petit-Yapo, Azaguié, Akébéfiat et Elevi). Quant aux travaux de pépinière, ils ont été menés à la station de recherche fruitière d'Azaguié $\left(5^{\circ} 33 \mathrm{~N}\right.$ et $\left.4^{\circ} 03 \mathrm{O}\right)$ et à la Station de recherche forestière de Sangoué $\left(06^{\circ} 17 \mathrm{LN}\right.$ et
2001 ; Kouamé et al., 2008). En outre, l'étude de leur composition chimique a été entamée par Herzog (1992). Mais concernant la domestication qui constitue une étape décisive vers la valorisation de ces espèces, très peu d'études ont été conduites. Aujourd'hui, avec l'explosion démographique, la menace de disparition des reliques forestières affecte également les espèces résiduelles de produits forestiers non ligneux qu'elles abritent. Cette situation montre l'urgence d'accroître la connaissance que les populations ont de ces espèces et d'envisager leur sauvegarde. Pour contribuer à la domestication et à la valorisation de ces espèces, une enquête ethnobotanique a été réalisée dans le Département d'Agboville (Sud-est). L'objectif poursuivi était de déterminer les espèces alimentaires encore présentes dans cette région fortement anthropisée et de mettre en collections celles menacées de disparition.

$05^{\circ} 31 \mathrm{LO}$ ) dans les Départements d'Agboville et d'Oumé respectivement. Le climat d'Azaguié est bimodal ; deux saisons de pluies (mars à juin et octobre à novembre) alternent avec deux saisons sèches (décembre à février et août à septembre). La pluviométrie moyenne annuelle est de $1270 \mathrm{~mm}$. Les sols sont ferralitiques, remaniés, fortement désaturés sur schiste. La végétation originelle est une forêt dense humide sempervirente (Anonyme, 1971). Aujourd'hui, fortement dégradée, cette forêt 
luxuriante jadis, a fait place à des formations secondaires, des cultures de rente (café, cacao, hévéa, bananes) et des jachères. Le deuxième site de l'étude, la station de recherche forestière de Sangoué, a abrité des travaux de pépinière (production de plants) et les essais de comportement en plantation (collection) des essences étudiées. Les conditions écologiques y sont similaires à celles d'Azaguié: deux saisons des pluies et deux saisons sèches, une pluviométrie moyenne annuelle de $1320 \mathrm{~mm}$, des sols ferralitiques fortement désaturés et une végétation de forêt dense humide semi décidue, elle aussi, très dégradée actuellement (Lecompte, 1990).
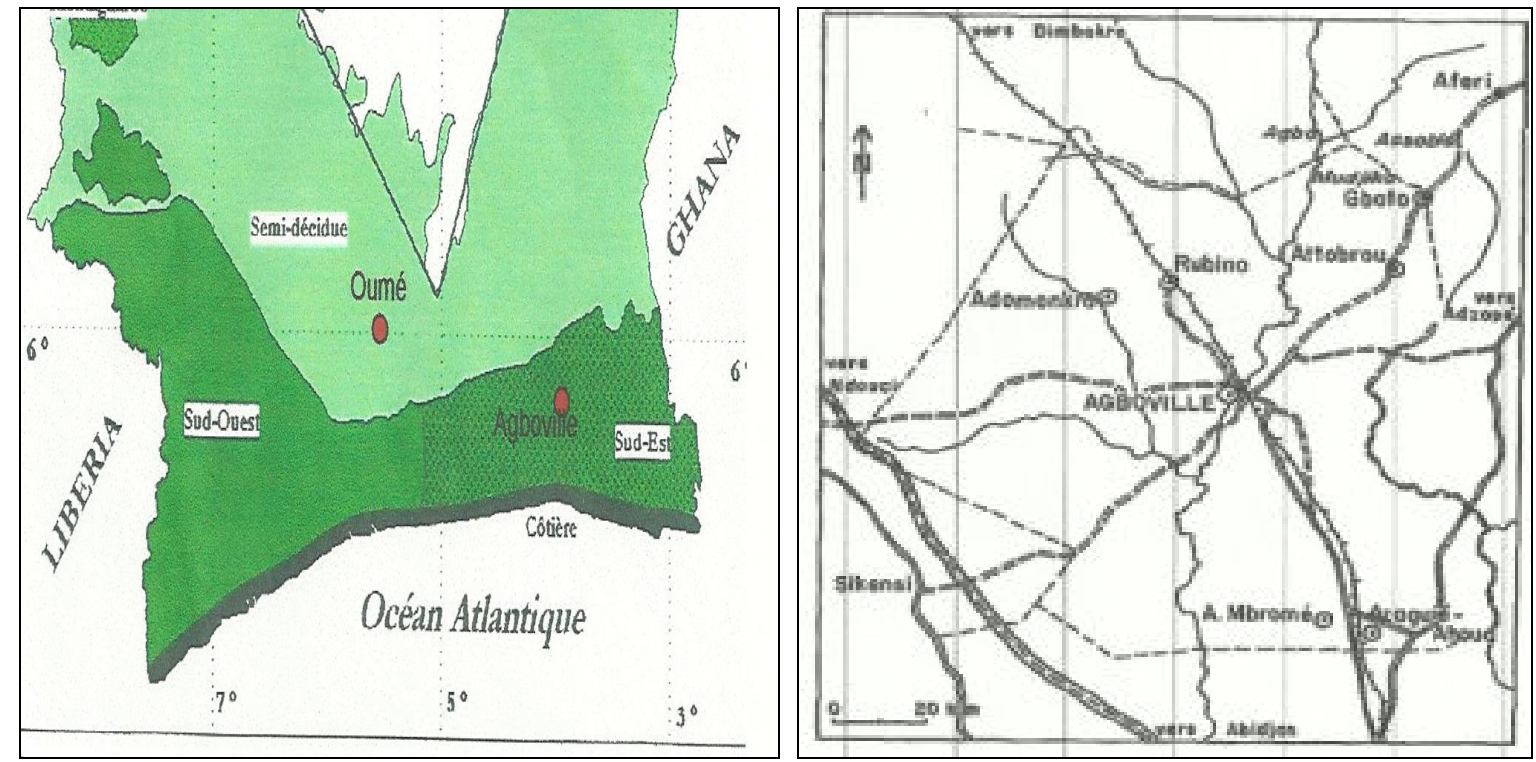

Figure 1 : Situation géographique des départements d'Agboville (Sud-Est) et d'Oumé (Centre-Ouest) en zone forestière de Côte d'Ivoire

Matériel végétal: Le matériel végétal étudié est constitué des espèces alimentaires (arbres, arbustes, lianes et herbes) inventoriées et récoltées dans le Département d'Agboville. Pour la production de plants en pépinière, nous avons utilisé des graines et des sauvageons de ces espèces.

\section{Méthode}

Enquête ethnobotanique: Pour l'enquête, les personnes retenues (60 au total) étaient des adultes âgés de 30 à 40 ans environ, sensées bien connaître les espèces fruitières sauvages de leur terroir. Pendant ces enquêtes faites d'interviews individuelles et collectives, les informations recherchées ont porté sur les noms locaux des espèces, les périodes de fructification, l'habitat, la forme biologique, les organes consommés et les autres usages. Dans chaque localité, la phase de questionnaire a été suivie d'une collecte de matériel végétal, dans le milieu naturel. Ainsi, des fruits et des graines de certaines espèces ont été collectés. Pour d'autres, ce sont des sauvageons qui ont été déterrés sous les semenciers, étant donné que la période de fructification de ces espèces était passée, au moment de l'enquête.
Détermination des espèces inventoriées : Les espèces ont été identifiées en utilisant la nomenclature de Hutchinson et Dalziel (1954-1972) ainsi que la Flore de Côte-d'Ivoire (Aké Assi, 2001). Suite à cette détermination, une flore des espèces fruitières sauvages du Département d'Agboville a été dressée.

Production de plants en pépinière : Des plants ont été produits en pépinière à Azaguié (Sud-Est). Avant le semis, les graines ont été extraites des fruits mûrs en phase de putréfaction. Elles ont été semées avec leur coque, sans prétraitement. Deux modes de reproduction ont été utilisés, selon le type de matériel végétal qui était disponible pour chaque espèce: semis de graines et repiquage de sauvageons dans des sachets de polyéthylène (Figure 2). Les sachets contenaient un substrat sain constitué de terre franche, de sable et de fumier (bouse de vache) en proportions égales. Par sachet, une graine a été enfouie dans le substrat préalablement mouillé, à environ $2 \mathrm{~cm}$ de profondeur. Ainsi, en pépinière, sept (7) espèces ont été reproduites par graines et six (6) autres par élevage de sauvageons. Pour les sauvageons, 10 à 130 plantules ont été repiquées par espèce et pour les semis, 10 à 60 graines 
ont été semées par espèce. Les pots ont été arrosés 3 à 4 fois par semaine. Les jeunes plants ont été entretenus (désherbage, arrosage, lutte contre les parasites) jusqu'à atteindre la hauteur de 30 à $40 \mathrm{~cm}$, leur permettant d'être transplantés au champ sans risque physiologique majeur. Les taux de levée et les durées de séjour en pépinière ont été observés.

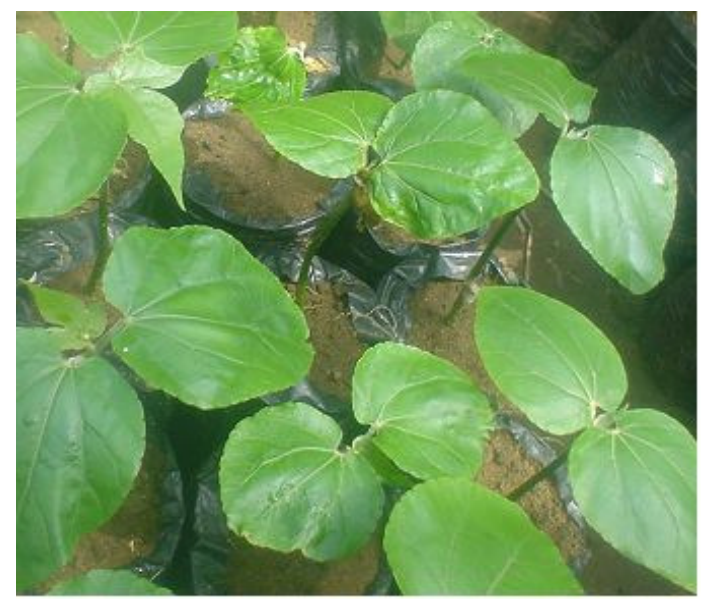

Figure 2 : Jeunes plants de Ricinodendron heudelotii en sachets de polyéthylène à la station d'Azaguié

Essai de comportement au champ (collection) $A$ Oumé, les graines ramenées de la prospection ont été semées dans des sachets en plastique contenant du terreau forestier tamisé. Les plants âgés de 6 à 12 mois, en pépinière, ont été transplantés au champ, à l'écartement de $4 \mathrm{~m} \mathrm{x} 4 \mathrm{~m}$, (625 pieds/ha) pendant la

\section{RESULTATS}

Espèces inventoriées: Dix-huit (18) espèces ont été recensées (Tableau 1). Elles se répartissent en 18 genres et 15 familles. Les familles les plus représentées sont les Anacardiaceae (3 espèces) et les Annonaceae (2 espèces). Les autres familles ne comportent qu'une seule espèce chacune. Les arbres constituent le type morphologique dominant $(81,8 \%)$, tandis que les lianes et les herbes $(4,54 \%$ chacune) sont les formes les plus rares (Figure 4). Les espèces à graines ou amandes comestibles représentent $50 \%$ des taxons rencontrés. grande saison des pluies (Figure 3). Dans cette localité, quatre des espèces inventoriées ayant un intérêt communautaire avéré ont été retenues pour le suivi du comportement en plantation (taux de survie, hauteur et fructification).

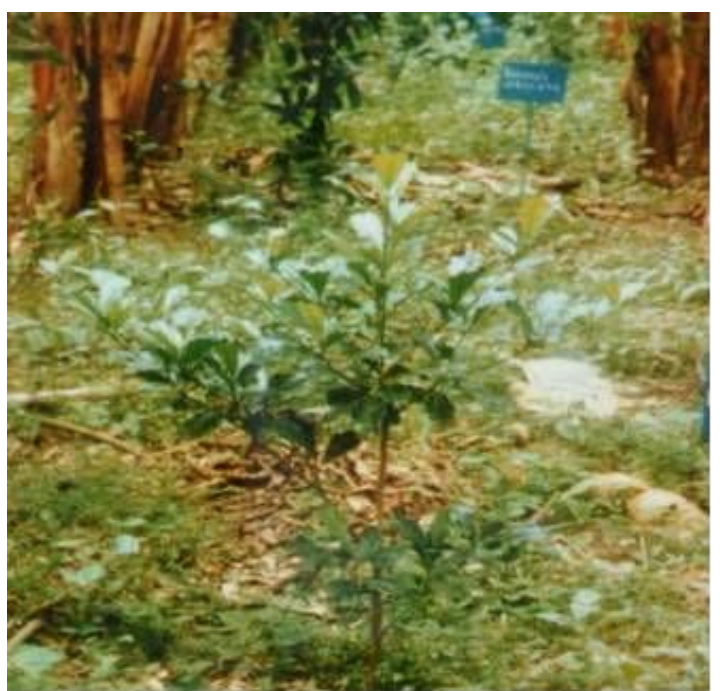

Figure 3: Quelques pieds de Makoré (Tieghemella heckelii ) en collection à la station d'Oumé

Analyses des données : Les données mesurées ont été saisies sur Excel et des statistiques descriptives ont été effectuées avec le logiciel Genstat.

Suivent, respectivement, celles produisant les fruits et les feuilles comestibles (45,4 et 5,54\%). Chez plusieurs de ces espèces, les graines, les feuilles, les écorces et les racines sont utilisées en pharmacopée traditionnelle. Concernant l'habitat, les espèces recensées sont majoritairement ubiquistes $(66,6 \%)$; elles se rencontrent en forêt, dans les jachères et dans les vergers de cacaoyers. Seules 33,3\% d'entre elles se rencontrent uniquement en forêt.

Tableau 1 : Flore des espèces recensées

\begin{tabular}{lcll}
\hline Espèce & Famille & Nom local & $\begin{array}{l}\text { Organes consomméset autres } \\
\text { usages }\end{array}$ \\
\hline Antrocaryon micraster & Anacardiaceae & Akwa (Abbey) & Les graines sont consommées \\
\hline
\end{tabular}




\begin{tabular}{|c|c|c|c|}
\hline Beilschmiedia mannii & Lauraceae & $\begin{array}{l}\text { Atiokouo (Abbey), Bitéi } \\
\text { (Centre-Ouest) }\end{array}$ & $\begin{array}{l}\text { Les cotylédons servent à } \\
\text { confectionner des sauces }\end{array}$ \\
\hline Buchholzia coriacea & Capparidaceae & Amon (Abbey) & Utilisé surtout en pharmacopée \\
\hline Cleistopholis patens & Annonaceae & $\begin{array}{l}\text { Bouhoubou (Dida),Sobou } \\
\text { (Abbey) }\end{array}$ & $\begin{array}{l}\text { L'amande de la graine sert à } \\
\text { aromatiser les sauces }\end{array}$ \\
\hline Coula edulis & Olacaceae & Attia (Abbey) & $\begin{array}{l}\text { L'amande de la graine, à goût de } \\
\text { noix est consomée }\end{array}$ \\
\hline Dacryodes klaineana & Burseraceae & $\begin{array}{l}\text { Krinja (Agni), Vi (Abbey) } \\
\text { Sagnon (Dida) }\end{array}$ & $\begin{array}{l}\text { Fruits consommés frais et feuilles } \\
\text { utilisées en pharmacopée }\end{array}$ \\
\hline Garcinia kola & Clusiaceae & $\begin{array}{l}\text { Attiokouo } \\
\text { Aouolié (Abbey) }\end{array}$ & $\begin{array}{l}\text { Graines amères consommées } \\
\text { pour leur effet stimulant }\end{array}$ \\
\hline Irvingia gabonensis & Irvingiaceae & $\begin{array}{l}\text { Boborou (Abbey), } \\
\text { Kakourou (Gouro) }\end{array}$ & $\begin{array}{l}\text { Les cotylédons servent à } \\
\text { confectionner des sauces }\end{array}$ \\
\hline Myrianthus arboreus & Cecropiaceae & $\begin{array}{l}\text { Wougnan (Abbey), } \\
\text { Pissia (Dida) } \\
\text { Djin (Attié) }\end{array}$ & $\begin{array}{l}\text { Les fruits sucrés acidulés sont } \\
\text { consommés } \\
\text { Les jeunes feuilles servent à la } \\
\text { confection de sauces }\end{array}$ \\
\hline $\begin{array}{l}\text { Ricinodendron } \\
\text { heudelotii }\end{array}$ & Euphorbiaceae & $\begin{array}{l}\text { Akpi (Abbey, Baoulé) } \\
\text { Gbakouè (Dida) }\end{array}$ & $\begin{array}{l}\text { L'amande sert à confectionner des } \\
\text { sauces }\end{array}$ \\
\hline Spondias mombin & Anacardiaceae & $\begin{array}{l}\text { N'gba (Abbey, Akyé), } \\
\text { Toto (Dida) }\end{array}$ & $\begin{array}{l}\text { Fruits consommés et feuilles } \\
\text { utilisées en pharmacopée }\end{array}$ \\
\hline Tarrietia utilis & Sterculiaceae & Niangon & Les graines sont consommées \\
\hline Tetrapleura tetraptera & Fabaceae & Essé Essé (Abbey) & $\begin{array}{l}\text { Le fruit entier sert à parfumer l'eau de } \\
\text { boisson }\end{array}$ \\
\hline $\begin{array}{l}\text { Thaumatococcus } \\
\text { daniellii }\end{array}$ & Marantaceae & $\begin{array}{l}\text { Angondro N'gna } \\
\text { (Agni, Baoulé) }\end{array}$ & $\begin{array}{l}\text { Le mucilage entourant la graine } \\
\text { est un édulcorant }\end{array}$ \\
\hline Tieghemella heckelii & Sapotaceae & Makoré & $\begin{array}{l}\text { L'amande séchée de la graine } \\
\text { donne du beurre de Makoré }\end{array}$ \\
\hline Treculia africana & Moraceae & $\begin{array}{l}\text { Bléblindou (Agni) } \\
\text { Noumou sou (Bété) }\end{array}$ & $\begin{array}{l}\text { La pulpe du syncarpe séchée est } \\
\text { utilisée en sauce comme } \\
\text { succédané de la viande. Les } \\
\text { graines grillées sont comestibles }\end{array}$ \\
\hline Tricoscypha arborea & Anacardiaceae & $\begin{array}{l}\text { Dao (Abbey), Alakpin } \\
\text { (Agni), }\end{array}$ & $\begin{array}{l}\text { Les fruits frais très sucrés sont } \\
\text { consommés }\end{array}$ \\
\hline Xylopia aethiopica & Annonaceae & Essin (Agni), Lilo (Bété) & $\begin{array}{l}\text { Les graines séchées servent de } \\
\text { condiment, succédané du poivre }\end{array}$ \\
\hline
\end{tabular}




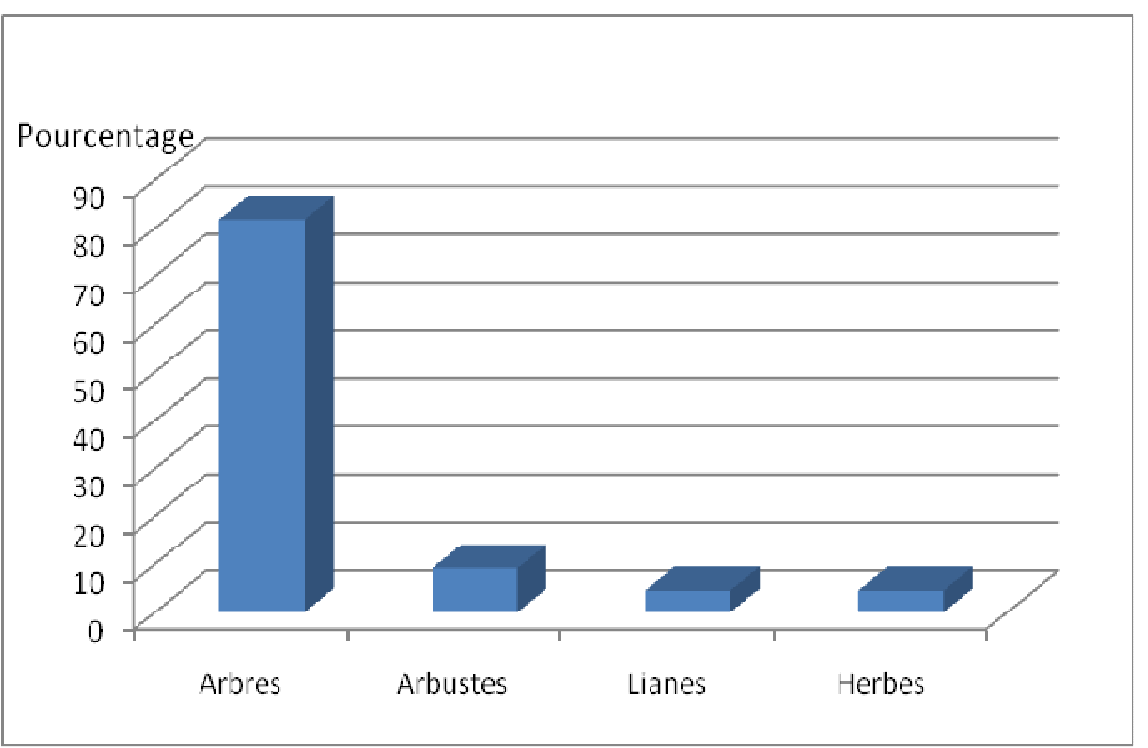

Figure 4 : Types morphologiques des espèces recensées

Taux de germination des graines et taux de reprises des sauvageons : Le tableau 2 montre que les espèces qui ont affiché les taux de germination les plus élevés sont Buchholzia coriacea (100\%), Tieghemella heckelii (92,95\%), Cleistopholis patens $(83,33 \%)$ et Ricinodendron heudelotii $(80,76)$. Myrianthus arboreus a montré un taux de germination moyen $(60 \%)$ tandis que Garcinia kola a présenté le taux le plus faible (20\%). Le suivi spécifique des semences de Ricinodendron heudelottii a révélé un début de germination à 34 jours après semis (JAS) et une durée de germination de 35 jours.

Tableau 2 : Taux de germination des essences reproduites par graines

\begin{tabular}{lccc}
\hline \multicolumn{1}{c}{ Espèce } & $\begin{array}{c}\text { Graines } \\
\text { semées }\end{array}$ & $\begin{array}{c}\text { Plants } \\
\text { produits }\end{array}$ & $\begin{array}{c}\text { Taux de } \\
\text { germination (\%) }\end{array}$ \\
\hline Buchholzia coriacea & 10 & 10 & 100 \\
Cleistopholis patens & 18 & 15 & 83,33 \\
Garcinia kola & 20 & 4 & 20 \\
Myrianthus arboreus & 60 & 36 & 60 \\
Tieghemella heckelii & 51 & 47 & 92,95 \\
Tricoscypha arborea & 12 & 8 & 66,66 \\
Ricinodendron heudelotii & 156 & 126 & 80,76 \\
\hline
\end{tabular}

Concernant les plants issus de sauvageons (Tableau 3), leurs taux de reprise ont oscillé entre 27,27\% (Xylopia aethiopica) et $100 \%$ (Treculia africana). En plus de Treculia africana, trois autres espèces ont affiché des taux de reprise élevés. Ce sont: Irvingia gabonensis $(96,15 \%)$ Beilschmiedia mannii $(80 \%)$ et Dacryodes klaineana $(72,41 \%)$. Le taux de reprise des sauvageons de Antrocaryon micraster a été moyen (56,47\%).

Tableau 3 : Taux de reprise des essences reproduites par sauvageons

\begin{tabular}{lccc}
\hline Espèce & Sauvageons repiqués & Plants produits & Taux de reprise (\%) \\
\hline Antrocaryon micraster & 62 & 35 & 56,47 \\
Beilschmiedia mannii & 70 & 56 & 80 \\
Dacryodes klaineana & 29 & 21 & 72,41 \\
Irvingia gabonensis & 29 & 21 & 96,15 \\
Treculia africana & 130 & 130 & 100 \\
Xylopia aethiopica & 11 & 3 & 27,27 \\
\hline
\end{tabular}


Durée de séjour en pépinière: L'étude conduite à Azaguié a permis de déterminer la durée de séjour en pépinière, à savoir le temps nécessaire à ces essences pour atteindre la hauteur de $30-40 \mathrm{~cm}$ permettant de les transplanter sans préjudice physiologique. Ainsi, deux durées de séjour en pépinière ont pu être distinguées ; la durée courte (3-6 mois) et la durée longue (8-11mois). Les différentes espèces étudiées et leurs durées de pépinière sont présentées au tableau 4. Hormis Irvingia gabonensis, Ricinodendron heudelotii et Trycoscypha arborea, les autres espèces de l'étude ont montré de longues durées de séjour en pépinière.

Tableau 4 : Durée de séjour en pépinière des essences en fonction du matériel végétal utilisé

\begin{tabular}{lcc}
\hline Durée de séjour & Durée courte (3-6 mois) & Durée longue (8-11 mois) \\
\hline Espèces issues de sauvageons & Irvingia gabonensis & Antrocaryon micraster \\
& & Beilschmiedia mannii \\
& & Dacryodes klaineana \\
& & Treculia africana \\
Espèces issues & Xylopia aethiopica \\
de graines & Buchholzia coriacea \\
& Ricinodendron heudelotii & Cleistopholis patens \\
& Tricoscypha arborea & Garcinia kola \\
& & Myrianthus arboreus \\
& Tieghemella heckelii \\
\hline
\end{tabular}

Croissance des essences en collection : La croissance des essences a été suivie en collection pendant les 7 premières années. $A 7$ ans, le taux de survie le plus élevé a été observé chez Spondias mombin (86\%) et le plus faible chez Irvingia gabonensis (40,70\%). Quant à Tieghemella heckelii (66\%) et Ricinodendron heudelottii $(55,10 \%)$, elles ont présenté des taux de survie moyens (Tableau 5).

Tableau 5 : Taux de survie des essences étudiées, en plantation

\begin{tabular}{lccc}
\hline Espèces & $\begin{array}{c}\text { Nombre de pieds } \\
\text { plantés }\end{array}$ & $\begin{array}{c}\text { Nombre de } \\
\text { pieds vivants }\end{array}$ & Taux de survie (\%) \\
\hline Tieghemella heckelii & 206 & 136 & 66 \\
Spondias mombin & 69 & 60 & 86 \\
Irvingia gabonensis & 86 & 35 & 40,70 \\
Ricinodendron heudelotii & 49 & 32 & 55,10 \\
\hline
\end{tabular}

A 7 ans, la croissance des espèces étudiées a révélé d'un côté, Ricinodendron heudelottii et Spondias mombin avec les plants les plus hauts $(12,61$ et $9,21 \mathrm{~m}$ respectivement) et de l'autre Irvingia gabonensis et Tieghemella heckelii ayant les plus petits sujets $(4,69 \mathrm{~m}$ et 2,93 m). L'évolution des hauteurs (Figure 5) montre que Ricinodendron heudelottii, Spondias mombin et Irvingia gabonensis ont présenté des rythmes de croissance presque identiques de 3 à 5 ans, avant de se différencier entre 5 et 7 ans. Les accroissements moyens ainsi enregistrés sont de 1,81 et $1,31 \mathrm{~m}^{-a^{-1}}$ chez Ricinodendron heudelottii et Spondias mombin alors qu'ils sont de 0,67 et 0,42 m.an-1 respectivement chez Irvingia gabonensis et Tieghemella heckelii. Concernant la fructification, Irvingia gabonensis et Ricinodendron heudelotii sont entrées en production, respectivement à 10 et 12 ans. 


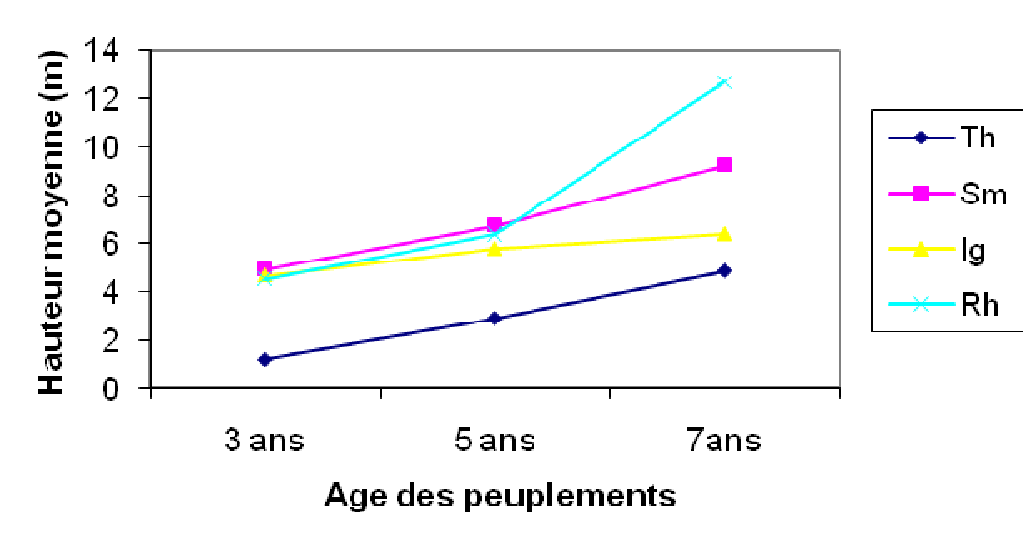

Figure 5: Evolution des hauteurs des essences étudiées entre 3 et 7 ans

Th: Tiegemella heckellii, Sm :Spondias mombin, Ig : Irvingia gabonnensis,

$R h$ : Ricinodendron heudelotii

\section{DISCUSSION}

Aspects floristiques : La flore de notre milieu étude, avec 18 espèces réparties en 18 genres et 15 familles, paraît réduite et peu diversifiée, comparée aux flores d'études similaires (Ambé, 2001 et Kouamé et al., 2008). Ces auteurs dont les travaux ont porté sur les régions du Nord-Ouest (zone de savane) d'une part et du CentreOuest forestier d'autre part, ont dévoilé des flores de 101 et 73 espèces respectivement. Bien que le nombre de personnes enquêtées et les superficies de végétation prospectées puissent différer d'une zone à une autre, les résultats actuels semblent indiquer une érosion végétale bien plus importante dans la zone prospectée au SudEst. En effet, la forte anthropisation du Sud-Est ivoirien (zone de départ de la culture du cacaoyer), a eu pour corollaire la transformation de l'espace forestier en mosaïque de bosquets appauvris et de jachères de Chromolaena odorata (Kouassi et al., 2009). Dans ces écosystèmes dégradés, la régénération naturelle des essences forestières est compromise voire inhibée non seulement par la raréfaction des semenciers mais aussi par le ramassage systématique des fruits comestibles. De même, les espèces herbacées et les lianes sont les plus rares parce que régulièrement défrichées lors de la création des plantations. En revanche, certaines essences arborées sont protégées dans les vergers en raison de leur compatibilité avec le cacaoyer et le caféier (Mollet et al., 2000). La présence de ces espèces dans les milieux anthropisés comme les jachères et les vergers de cultures de rente dénote de la protection dont elles sont l'objet de la part des paysans. Dans la plupart des études portant sur les espèces fruitières sauvages, en effet, plusieurs espèces survivent dans des milieux fortement anthropisés parce qu'elles font l'objet de protection et même de replantation de la part de certains paysans (Tchatat et Ndoye, 2006). Les paysans concernés montrent ainsi un début de prise de conscience sur l'importance socioéconomique de ces produits et les désagréments que leur disparition peut engendrer.

Production de plants en pépinière: Onze (11) des treize (13) espèces étudiées ont montré des taux de germination ou de reprise des sauvageons de plus de $50 \%$. Ces résultats indiquent que la plupart des taxons étudiés ont de bonnes aptitudes à la reproduction en milieu contrôlé et donc à la domestication. La germination de Garcinia kola et de Xylopia aethiopica a montré dans d'autres études, à Anguédédou (Sud de la Côte d'Ivoire) des difficultés liées à la longue période de levée de dormance au cours de laquelle plusieurs semences ont perdu leur faculté germinative par putréfaction (CNRA, 2010). Concernant la longue durée de séjour en pépinière (8-11 mois) qui semble caractériser la majorité des ces essences, elle résulte de la longueur de la période de dormance de certaines graines mais aussi du faible rythme de croissance en hauteur des jeunes plants. Généralement, la forte dormance des graines est due à la présence de téguments imperméables à l'eau et à l'air qui peuvent différer la germination pendant plusieurs mois ou années (Danthu et al., 2003). Dans le cas spécifique des graines de Ricinodendron heudelotii qui ont germé à 34 JAS dans la présence étude (sans prétraitement), les travaux de Kouamé et al. (2012) ont montré qu'un simple trempage dans de l'eau courante a permis de ramener le délai de germination à 12-14 jours. L'absence de prétraitement des graines utilisées à Azaguié peut ainsi justifier les longues durées de séjour en pépinière. 
Les essences en collection : L'utilisation des produits forestiers non ligneux (PFNL) fait partie essentiellement du secteur informel; c'est pourquoi peu de données fiables sont disponibles en ce qui concerne la production, le commerce et le nombre de personnes impliquées dans ce secteur (Vantomme et Gazza, 2010). II en est de même pour les données sur la croissance de ces essences en parcelle de domestication (collections). Par conséquent, c'est en référence à certaines espèces forestières utilisées dans les opérations de reboisement en Côte d'lvoire que nous avons analysé le comportement en collection des essences étudiées. Les fruitiers sauvages de la présente étude peuvent être classés dans la catégorie des espèces forestières à croissance initiale moyenne. En effet Dupuy (1986) désigne comme telles les essences forestières (Niangon, Okoumé et Sipo) qui ont des rythmes de croissance de

\section{CONCLUSION ET PERSPECTIVES}

La présente étude a mis en lumière une flore restreinte d'espèces arborées majoritairement, résultat de la forte anthropisation du milieu exploré. Les essences révélées ont montré de bonnes aptitudes à la régénération par graines et par sauvageons ainsi qu'une bonne adaptation à la conduite en peuplement. La conduite de sauvageons en pépinière peut aider à résoudre les problèmes liés à la rareté saisonnière des semences. Par leur croissance initiale satisfaisante, ces essences sont aptes à se développer dans les systèmes agroforestiers traditionnels associant les arbres aux vivriers ou aux cultures de rente (café et cacao). II importe, toutefois, d'appliquer des prétraitements aux semences de ces espèces pour lever
0,5 à $1,2 \mathrm{~m} \mathrm{an}^{-1}$. Cet auteur a observé chez le Niangon (Tarieta utilis) et l'Okoumé (Aucoumea klaineana) des accroissements moyens respectifs de 1,12 et 0,94 m.an-1, à 5 ans, en zone forestière de Côte d'Ivoire. Cette bonne croissance initiale dispose ces essences à résister à la compétition des adventices telles que Chromolaena odorata qui est prépondérante dans la zone d'introduction. Ces performances les rendent aptes à l'association avec les cultures de rente (café et cacao) qui croissent à des rythmes quasiment similaires durant les premières années. Quant aux taux de survie, ils sont moyens à élevés (40,7 à $86 \%$ ) et dénotent d'une bonne adaptation de ces essences à la zone d'introduction. Par ces deux critères (taux de survie élevés et bonnes croissance initiale), les espèces étudiées montrent une bonne aptitude à la domestication et à l'introduction dans les systèmes de cultures traditionnels.

plus rapidement la dormance et réduire leurs durées de séjour en pépinière. De même, il est à envisager la production de plants des espèces prioritaires par la multiplication végétative. Ceci permettra de hâter leur entrée en production et de susciter un plus grand intérêt de la part des populations rurales. II convient en outre, de conduire des études agronomiques (essai de densité, besoins hydriques et nutritionnels, sensibilité aux maladies et aux ravageurs, etc.) afin de définir des itinéraires techniques fiables pour faire de ces espèces sauvages des cultures de rente, à terme.

\section{REMERCIEMENTS}

Les auteurs remercient l'Association Ivoirienne des Sciences Agronomique (AISA) pour le financement de la présente étude réalisée dans le cadre des Projets d'Etudes Participatives (PEP).

\section{REFERENCES}

Aké-Assi L. 2001. Flore de la Côte d'lvoire. Vol. 1, catalogue, systématique, biogéographie et écologie. Conservatoire et Jardin Botanique, Genève, Switzerland, Boissera $57,396 \mathrm{p}$.

Ambé G.A. 2001. Les fruits sauvages comestibles des savanes guinéennes de Côte d'Ivoire: état de la connaissance par une population locale, les Malinkés. Biotechnol. Agron. Soc. Environ. 5(1) : 43-58.

Anonyme 1971. Le milieu naturel de la Côte d'Ivoire. Document ORSTOM N50. IRD Editions. $460 \mathrm{p}$.

Aubréville A.1959. Flore forestière de Côte d'ivoire. 2ème Ed. C.T.F.T. Nogent sur Marne. Vol.3. 334 p.
CNRA 2010. Rapport d'activités 2010.Programme Forêt et Environnement. CNRA Abidjan. $19 \mathrm{p}$.

Danthu P., Roussel J., Neffati M.2003. La graine et la germination d'Acacia raddiana, un arbre au désert: 265 283.http://www.horizon.documentation.ird.fr /exldoc/pleins_texte/divers.10-07/010033306 pdf.Consulté le 15/05/2013

Dupriez H., De Leener Ph. 1993. Arbres et agricultures multiétagées d'Afrique. Terre et vie. CTA. Wageningen. $280 \mathrm{p}$.

Dupuy B. 1986. Principales règles de sylviculture pour les espèces à vocation bois d'œurre. Rapport 
Centre Technique Forestier Tropical. (CTFT) Abidjan. Côte d'Ivoire, $112 \mathrm{p}$.

Gautier-Beguin D.1992. Etude ethnobotanique des plantes de cueillette à utilisation alimentaire dans un village au sud du V-Baoulé (Côte d'Ivoire). Thèse de Doctorat. Université de Genève, $368 \mathrm{p}$.

Herzog F.M. 1992. Etude biochimique et nutritionnelle des plantes alimentaires sauvages dans le Sud du V-Baoulé, Côte d'Ivoire. Thèse Ecole Polytechnique Fédérale de Zurich (Suisse). 121 p.

Hutchinson J., Dalziel J. M., 1954-1972. Flora of West

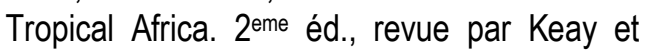
Hepper. Londres, Royaume-Uni, Crown Agents for Oversea Governments, 3 vol.

Kouamé N.M.T., Gnahoua G.M. 2008. Arbres et lianes spontanées alimentaires de Gagnoa (Centre Ouest de la Côte d'Ivoire). Bois et Forêts des Tropiques N² 298 (4) : 65-75.

Kouamé M.Th., Gnahoua G.M., Konan E. K., Traoré D. 2008. Les plantes spontanées alimentaires de la Région du Fromager (Gagnoa) : Flore, habitats et organes consommés. Sciences \& Nature Vol. $5 \mathrm{~N}^{\circ} 1: 61-70$.

Kouamé N.M.T., Gnahoua G.M., Mangara A. 2012. Essai de domestication de Ricinodendron heudelotii dans la région du Fromager (Côte d'lvoire). Journal of Applied Biosciences 56 : 4133-4141.

Kouassi K.H., Nguessan K., Kouassi Konan E. 2009. Flore post-culturale en zone de forêt dense semi décidue de Côte d'Ivoire. Journal of Applied Biosciences 19: $1026-1040$.

Lecomte Ph. (1990). Place et intégration de l'arbre dans l'exploitation agricole ivoirienne du Centre Ouest. Cas de la région d'Oumé. Mémoire CNEARC, Montpellier, France, $97 \mathrm{p}$.

Mollet M., Téré H., Herzog F. 2000. Ligneux à usages multiples dans les systèmes agraires tropicaux: une étude de cas de Côte d'Ivoire. Schweiz. Z. Forstwes., 151, 355-364.

Tchatat M., Ndoye O. 2006. Etude des Produits Forestiers Non ligneux d'Afrique Centrale: réalités et perspectives. Bois et Forêts des Tropiques. №289 (60) :27-35

Vantomme P., Gazza, S. 2010. Le défi de la sylviculture en faveur des Produits Forestiers Non Ligneux: de la cueillette à l'agriculture? Bois et Forêts des Tropiques. Nº304 (2) :5-13. 